\title{
A SHORT PROOF OF A THEOREM ON COMPOSITIONS OF D.C. MAPPINGS
}

\author{
LIBOR VESELÝ
}

(Communicated by R. Daniel Mauldin)

\begin{abstract}
In this note, there is presented an essentially shorter proof of the theorem that a composition of d.c. mappings is locally d.c., proved for finite dimensions by P. Hartman in 1959.
\end{abstract}

A mapping $F=\left(F_{1}, F_{2}, \ldots, F_{n}\right)$ from an open convex set $A \subset \mathbf{R}^{k}$ into $\mathbf{R}^{n}$ is called d.c. if each of its components $F_{j}$ is representable as a difference of two convex functions on $A$. P. Hartman proved that the composition of d.c. mappings is locally d.c. in the sense that every point in $A$ has a convex open neighborhood, on which the composite mapping is d.c. (see $[\mathbf{1},(\mathrm{II})]$ ).

In this paper, we give a simpler and much shorter proof of this fact. To prove a rather more general version of Hartman's theorem, we state the following definition. (All linear spaces in this note are real linear spaces. The continuous dual of a normed linear space $X$ is denoted by $X^{*}$.)

DEFINITION. Let $X, Y$ be normed linear spaces and let $A \subset X$ be a nonempty open convex set. A mapping $F: A \rightarrow Y$ is said to be d.c. on $A$ iff $F$ is continuous and there exists a continuous convex function $f$ on $A$ such that for any $y^{*}$ from a unit sphere in $Y^{*}$ the function $y^{*} \circ F+f$ is convex on $A$. (We shall say that $F$ is d.c. on $A$ with convex function $f$.)

It is easy to see that this definition is equivalent to the one written above, in case $X=\mathbf{R}^{k}, Y=\mathbf{R}^{n}$.

For a continuous convex function $f$ on an open convex subset $A$ of a normed linear space $X$ and for $x_{0} \in A$ we denote $\partial f\left(x_{0}\right)=\left\{x^{*} \in X^{*}: f(x)-f\left(x_{0}\right) \geq\left\langle x-x_{0}, x^{*}\right\rangle\right.$ for any $x \in A\}$. It is a well-known fact that this set (called a subdifferential of $f$ at $x_{0}$ ) is always nonempty (see $\left.[2, \S 43]\right)$.

THEOREM. Let $X, Y$ be Banach spaces and let $Z$ be a normed linear space. Let $A \subset X, B \subset Y$ be nonempty open convex sets. Let a mapping $F: A \rightarrow Y$ be d.c. on $A$ and $F(A) \subset B$. Let $G: B \rightarrow Z$ be d.c. and locally Lipschitz on $B$. Then $G \circ F$ is locally d.c. on $A$.

Note that in case $Z=\mathbf{R}^{m}$, it is possible to omit the assumption of local Lipschitz property of $G$ as it is fulfilled automatically.

PrOOF. Let $F$ ( $G$, respectively) be d.c. on $A$ (on $B$, resp.) with convex function $f$ ( $g$, resp.). Let $a_{0} \in A$ be an arbitrary point. Let $V \subset B$ be an open convex neighborhood of $F\left(a_{0}\right)$ such that both $G, g$ are Lipschitz on $V$ (with constants $\left.L_{G}, L_{g}\right)$ and let $U \subset A$ be an open convex neighborhood of $a_{0}$ with $F(U) \subset V$.

Received by the editors August 19, 1986.

1980 Mathematics Subject Classification (1985 Revision). Primary 26B35, 47H99; Secondary 26B25, 26B40. 
Denote $M=L_{G}+L_{g}$. We shall show that $G \circ F$ is d.c. on $U$ with convex function $g \circ F+M f$. Let $x_{0} \in U$.

(i) Let $x^{*} \in \partial f\left(x_{0}\right), y^{*} \in \partial g\left(F\left(x_{0}\right)\right)$ and let $y_{0}^{*} \in Y^{*}$ be such that $\left\|y_{0}^{*}\right\|=1$ and $y^{*}=\left\|y^{*}\right\| y_{0}^{*}$. Let $u^{*} \in \partial\left(y_{0}^{*} \circ F+f\right)\left(x_{0}\right)$. Then any $x \in U$ satisfies

$$
\begin{aligned}
& g(F(x))+M f(x)-\left[g\left(F\left(x_{0}\right)\right)+M f\left(x_{0}\right)\right] \\
& \quad \geq\left\langle F(x)-F\left(x_{0}\right), y^{*}\right\rangle+M\left[f(x)-f\left(x_{0}\right)\right] \\
& \quad=\left\|y^{*}\right\|\left[\left\langle F(x)-F\left(x_{0}\right), y_{0}^{*}\right\rangle+f(x)-f\left(x_{0}\right)\right]+\left(M-\left\|y^{*}\right\|\right)\left[f(x)-f\left(x_{0}\right)\right] \\
& \quad \geq\left\|y^{*}\right\|\left\langle x-x_{0}, u^{*}\right\rangle+\left(M-\left\|y^{*}\right\|\right)\left\langle x-x_{0}, x^{*}\right\rangle,
\end{aligned}
$$

since $\left\|y^{*}\right\| \leq L_{g} \leq M$. This means that the function $g \circ F+M f$ has a continuous support at an arbitrary point $x_{0} \in U$. Hence this function is continuous and convex on $U$ (see [2, Theorem 43C]).

(ii) Let $z^{*} \in Z^{*}$ satisfy $\left\|z^{*}\right\|=1$, let $x^{*} \in \partial f\left(x_{0}\right), v^{*} \in \partial\left(z^{*} \circ G+g\right)\left(F\left(x_{0}\right)\right)$, and let $v_{0}^{*} \in Y^{*}$ be such that $\left\|v_{0}^{*}\right\|=1$ and $v^{*}=\left\|v^{*}\right\| v_{0}$. Let $w^{*} \in \partial\left(v_{0}^{*} \circ F+f\right)\left(x_{0}\right)$. Then $\left\|v^{*}\right\| \leq M$ and the following inequalities hold for any $x \in U$ :

$$
\begin{aligned}
& \left\langle G(f(x)), z^{*}\right\rangle+g(F(x))+M f(x)-\left[\left\langle G\left(F\left(x_{0}\right)\right), z^{*}\right\rangle+g\left(F\left(x_{0}\right)\right)+M f\left(x_{0}\right)\right] \\
& \quad \geq\left\langle F(x)-F\left(x_{0}\right), v^{*}\right\rangle+\left\|v^{*}\right\|\left[f(x)-f\left(x_{0}\right)\right]+\left(M-\left\|v^{*}\right\|\right)\left[f(x)-f\left(x_{0}\right)\right] \\
& \quad \geq\left\|v^{*}\right\|\left\langle x-x_{0}, w^{*}\right\rangle+\left(M-\left\|v^{*}\right\|\right)\left\langle x-x_{0}, x^{*}\right\rangle .
\end{aligned}
$$

The function $z^{*} \circ G \circ F+g \circ F+M f$ is continuous and convex on $U$ by the same argument as in (i). The theorem is proved.

\section{REFERENCES}

1. P. Hartman, On functions representable as a difference of convex functions, Pacific J. Math. 9 (1959), 707-713.

2. A. W. Roberts and D. E. Varberg, Convex functions, Academic Press, New York and London, 1973.

Universita Karlova, Matematicko-Fysikální Fakulta, KMA, Sokolovská 83, 18600 Praha 8, CZECHOSLOVAKIA 